\title{
The effect of the physical characteristics of cues and targets on facilitation and inhibition
}

\author{
JAY PRATT \\ University of Toronto, Toronto, Ontario, Canada \\ JAMIE HILLIS \\ University of California, Berkeley, California \\ and \\ JASON M. GOLD \\ University of Toronto, Toronto, Ontario, Canada
}

\begin{abstract}
The present experiment was conducted in order to examine the role of cue-target discriminability on early occurring attentional cuing effects and late occurring inhibition of return (IOR). The experiment used a single target stimulus in conjunction with three different cue stimuli. The cues were the same as the target, different in color, shape, and luminance to the target, or did not spatially overlap with the target. At shorter stimulus onset asynchronies (SOAs; 100 and $200 \mathrm{msec}$ ), attentional cuing effects were only found with the nonoverlapping cues. However, at longer SOAs (400 and $800 \mathrm{msec}$ ), approximately equal IOR effects were found with all three types of cues. The results indicated that the physical characteristics of the cues and targets affected the pattern of reaction times at the shorter SOAs but not at the longer SOAs. The conclusion is that the biphasic pattern of early facilitation and late inhibition following a peripheral cue should not be considered the definitive signature of the peripheral cuing paradigm.
\end{abstract}

A peripheral visual cue, such as a flashed spot of light, has two measurable effects on human responses to a subsequent target. First, in a short interval immediately following the cue (less than $300 \mathrm{msec}$ ), targets are detected faster at cued than at uncued locations (e.g., Posner, 1980). Second, if the time interval between the cue and target is greater than $300 \mathrm{msec}$, responses to the cued location become slower than to uncued locations. These two effects have been explained in terms of attentional orienting. The early faster response is generally thought to be due to the shifting of attention to the cued location prior to the presentation of the target (e.g., Posner, 1980). The attentional cuing effect has been used to examine the operation of the attentional system in a wide variety of situations (e.g., Egly \& Homa, 1991; Folk, Remingtion, \& Johnston, 1992). The later slower response at the cued location is usually attributed to inhibition of return (IOR), a mechanism typically thought to inhibit attention from returning to previously attended (i.e., cued) locations ${ }^{1}$ (e.g., Posner \& Cohen, 1984; Pratt, 1995; Tipper, Weaver,

This research was supported by operating and equipment grants from the Natural Sciences and Engineering Council of Canada to J.P. The authors thank the editor and three anonymous reviewers for their very helpful comments regarding this paper. Correspondence concerning this article should be addressed to J. Pratt, Department of Psychology, 100 St. George Street, University of Toronto, Toronto, ON M5S 3G3 Canada (e-mail: pratt@psych.utoronto.ca).
Jerreat, \& Burak, 1994). The issue addressed in the present experiment is whether the biphasic pattern of response times in peripheral cuing experiments is obligatory or whether IOR can occur without early attentional cuing effects.

Maylor (1985) was the first to explicitly examine the relationship between attentional cuing effects and IOR effects. She reasoned that, if IOR is due to the prior orientation of attention to a peripheral location, secondary tasks that eliminate early attentional cuing effects should also eliminate late IOR effects. Using various eye movement secondary tasks, she did indeed find that IOR did not occur unless preceded by attentional cuing effects. This experiment, supplemented by the many IOR studies that have shown preceding attentional cuing effects (e.g., Posner \& Cohen, 1984; Pratt \& Abrams, 1999), has led to the conclusion that the biphasic pattern of response times is the signature of the peripheral cuing paradigm. The extension of this notion is that any late occurring inhibition following a cue can only be considered IOR if it is preceded by early facilitation. The pervasiveness of this notion can be seen in the IOR studies that have specifically included short stimulus onset ashynchronies (SOAs) in order to show the required attentional cuing effects (e.g., Pratt \& Abrams, 1995; Rafal, Calabresi, Brennan, \& Sciolto, 1989). In addition, recent studies commenting on earlier IOR research have, on the basis of the failure to find attentional cuing effects at short SOAs, questioned 
whether the late inhibitory effects found in the earlier research should be considered IOR (e.g., Taylor \& Klein, 1998a).

Although there are many studies that have found attentional cuing effects followed by IOR, the research evidence for the biphasic pattern of response times is not unanimous. In a series of experiments, Tassinari and his colleagues (Tassinari, Agliotti, Chelazzi, Peru, \& Berlucchi, 1994; Tassinari \& Berlucchi, 1993) consistently found either no advantage for targets at cued locations with short (less than $300 \mathrm{msec}$ ) SOAs or slower responses for targets at the cued locations. However, they also consistently found inhibition at the longer SOAs. Although the finding of inhibition at short SOAs is unique to the methods used by Tassinari and colleagues, Danziger, Kingstone, and Snyder (1998) and Enns and Richards (1997) have also reported no facilitatory effects at short SOAs. Thus, there is some evidence to suggest that the biphasic pattern of facilitation and inhibition is not always a consequence of attentional orienting.

Recently, there have been two attempts to explain why Tassinari and his colleagues did not find that attentional cuing effects precede IOR effects. Maruff, Yucel, Dankert, Stuart, and Currie (1999) focussed on the temporal overlap between cues and targets. They found attentional cuing effects only when the cue overlapped in time with the target and IOR only when the cue did not overlap with the target. Moreover, the duration of the target interacted with the duration of the cue, so that early and late inhibition were found with short duration $(50-\mathrm{msec})$ cues and targets. In addition, null effects at the short SOA were found when short duration cues were combined with long duration targets (visible until response). From these results, Maruff et al. concluded that the temporal characteristics of the cues and targets play a major role in determining whether attentional cuing effects and/or IOR effects will be found. However, there are numerous examples in the literature in which early facilitation and late IOR have been found when the cues did not overlap with targets at any SOA (e.g., Lupiáñez, Milán, Tornay, Madrid, \& Tudela, 1997; Maylor, 1985; Pratt \& Abrams, 1995).

In trying to explain the results found by Tassinari et al. (1994), Lupiáñez and Weaver (1998) focussed on the discriminability between cues and targets. Specifically, they noted that, at the shortest SOA used by Tassinari et al. (1994) $(0 \mathrm{msec})$, the task was no longer a detection task but a go-no go discrimination task in which both cue and target occurred at the same location. When the cue and target occur at the same location with $\mathrm{SOA}=0$, the discrimination between cue and target would be very difficult. This discrimination was important because there were catch trials in which the cue appeared without the target. However, the discrimination between cue and target was not difficult when they occurred in different spatial locations. Therefore the longer response times for $\mathrm{SOA}=0$, when the cue and target occurred in the same location (which created the appearance of an early inhibitory effect), may have been a result of difficulty in discriminating the cue from the target. However, Tassinari,
Aglioti, Chelazzi, Peru, and Berlucchi (1998) have responded to the comments of Lupiáñez and Weaver by providing new evidence of early inhibition in a paradigm that did not use catch trials and therefore was clearly a detection task and not a go-no go discrimination task. Moreover, Tassinari, Aglioti, Chelazzi, Marzi, and Berlucchi (1987) had previously found early inhibitory effects with a $200-\mathrm{msec}$ SOA.

The present experiment was designed to further examine the role of cue-target discriminability on attentional cuing effects and IOR. In the experiment described below, we have manipulated cue-target discriminability with differences in color, shape, luminance, and in spatial overlap. One target stimulus (a white filled-in box) was used in conjunction with three different types of cues: (1) a filled-in box of the same shape, color, and luminance as the target (same condition); (2) a filled-in circle with a different shape, color, and luminance than the target (different condition); and (3) an outline of a box with the same color and luminance of the target that did not spatially or temporally overlap with the target (nonoverlap condition). Posner and Cohen (1984), in their original investigation of IOR, also used cues and targets that did not spatially overlap and found early facilitation and late IOR. It should also be noted that, similar to other IOR experiments we have been involved in (e.g., Pratt \& Abrams, 1995, 1999), the cues in the present experiment do not temporally overlap with the targets.

Given the purposes of the experiment and the three experimental conditions, some predictions can be made. If cue-target discriminability does affect responses at short SOAs, smaller attentional cuing effects should be found in the same condition as in the different or nonoverlap conditions. Although there is a lack of research on which to base predictions regarding the role of discriminability in IOR, it is possible to make predictions regarding the relationship between attentional cuing effects and IOR. If early facilitation is a necessary precursor of IOR, any condition that does not yield an attentional cuing effect should also not yield an IOR effect. However, if the three experimental conditions produce different sized attentional cuing effects but same sized IOR effects (or vice versa), the biphasic pattern of early facilitation and late inhibition should not be regarded as a strict signature of the peripheral cuing paradigm. Finally, the lack of temporal overlap at both shorter and longer SOAs, in conjunction with long duration targets, should, according to Maruff et al. (1999), yield null effects at the shorter SOAs and IOR at the longer SOAs. Note that this prediction really only applies to the nonoverlap condition, which is very similar to the spatial arrangement of placeholders, cues, and targets used by Maruff et al.

\section{METHOD}

\section{Participants}

Ten undergraduate students from the University of Toronto participated in the experiment in exchange for course credit. All of the students were naive as to the purpose of the experiment. 


\section{Apparatus and Procedure}

The experiment was conducted in a dimly lit room using a PCcompatible computer. The participants were seated at a table directly in front of a computer monitor and their heads were held steady with a chin/head rest. The viewing distance was $44 \mathrm{~cm}$. A computer keyboard was placed on the table directly in front of the participants so that they could easily reach the keys.

The trial sequence is shown in Figure 1. Each trial began with a display consisting of a central fixation point and a place-holder box on each side presented in white (IBM color $7, \mathrm{~cd} / \mathrm{m}^{2}=30$ ) on a black background (IBM color $0, \mathrm{~cd} / \mathrm{m}^{2}=0$ ). The distance between the fixation point and the center of each box was $7^{\circ}$ of visual angle and each box subtended $1^{\circ}$. This display was presented for $1,500 \mathrm{msec}$, followed by a cue that was presented for $50 \mathrm{msec}$ and then removed. Following the offset of the cue, one of four delay periods occurred $(50,150,350$, or $750 \mathrm{msec}$, providing SOAs of 100, 200, 400, and $800 \mathrm{msec}$, respectively). Following the delay period, one of the boxes was completely filled in with white (IBM color $7, \mathrm{~cd} / \mathrm{m}^{2}=$ 30 ); this served as the target stimulus. Although only one target stimulus was used in this experiment, three types of cue stimuli were used. In the same condition, the cue was a white (IBM color 7, $\mathrm{cd} / \mathrm{m}^{2}=30$ ) filled in box subtending $0.9^{\circ}$. In the different condition, a filled in circle with a diameter of $0.9^{\circ}$ was used, and this cue had both a different color (green, IBM color 4$)$ and luminance $\left(\mathrm{cd} / \mathrm{m}^{2}=\right.$ 59) than the target. In the nonoverlap condition, the cue consisted of the outline of a box, subtending $1.1^{\circ}$ and centered at the same point as the place-holder box, that was presented in the same color as the target. In all conditions, the participants were instructed to press the " $z$ " key with their left hand if the target appeared in the left box or the "/" key with their right hand if the target appeared in the right box. They were also instructed to make the keypress as quickly and as accurately as possible. The target remained visible until one of the keys was pressed or 1,000 msec had elapsed. Keypress reaction times
(RTs) of less than $100 \mathrm{msec}$ or more than $1,000 \mathrm{msec}$ were considered errors. A brief tone was sounded when an error occurred.

\section{Design}

The experiment consisted of a total of 480 trials. The cue condition (same, different, and nonoverlap) was blocked (160 trials each) within each participant, and the order of the blocks was randomized between participants. With each block, the locations of the cue and the target were randomized. Thus, on half the trials, the target appeared in the same location as the cue (cued condition) and on the other half in the location opposite that of the cue (uncued condition). Also randomized within each of the blocks were the four possible interstimulus intervals (ISIs).

\section{Results}

Before the means were analyzed, error trials were removed, and trials which had RTs above or below 2.75 standard deviations $(S D \mathrm{~s})$ of the mean for that condition were also removed. Overall, only 120 trials (78 error trials and 42 trimmed trials) were removed across all participants $(2.1 \%$ of the total data set). The mean RT for each condition was then recalculated and are shown in Table 1 . The data were analyzed with a 4 (SOA: 100, $200,400,800) \times 2$ (trial type: cued or uncued) $\times 3$ (cue: same, different, or nonoverlap) analysis of variance (ANOVA). Main effects were found for SOA $[F(3,33)=$ $32.1, p<.0001]$, with faster RTs in the long condition, and for trial type $[F(1,11)=7.5, p<.02]$, with slower RTs for cued location. The two-way interactions between SOA and trial type $[F(3,33)=24.2, p<.0001]$ and be-

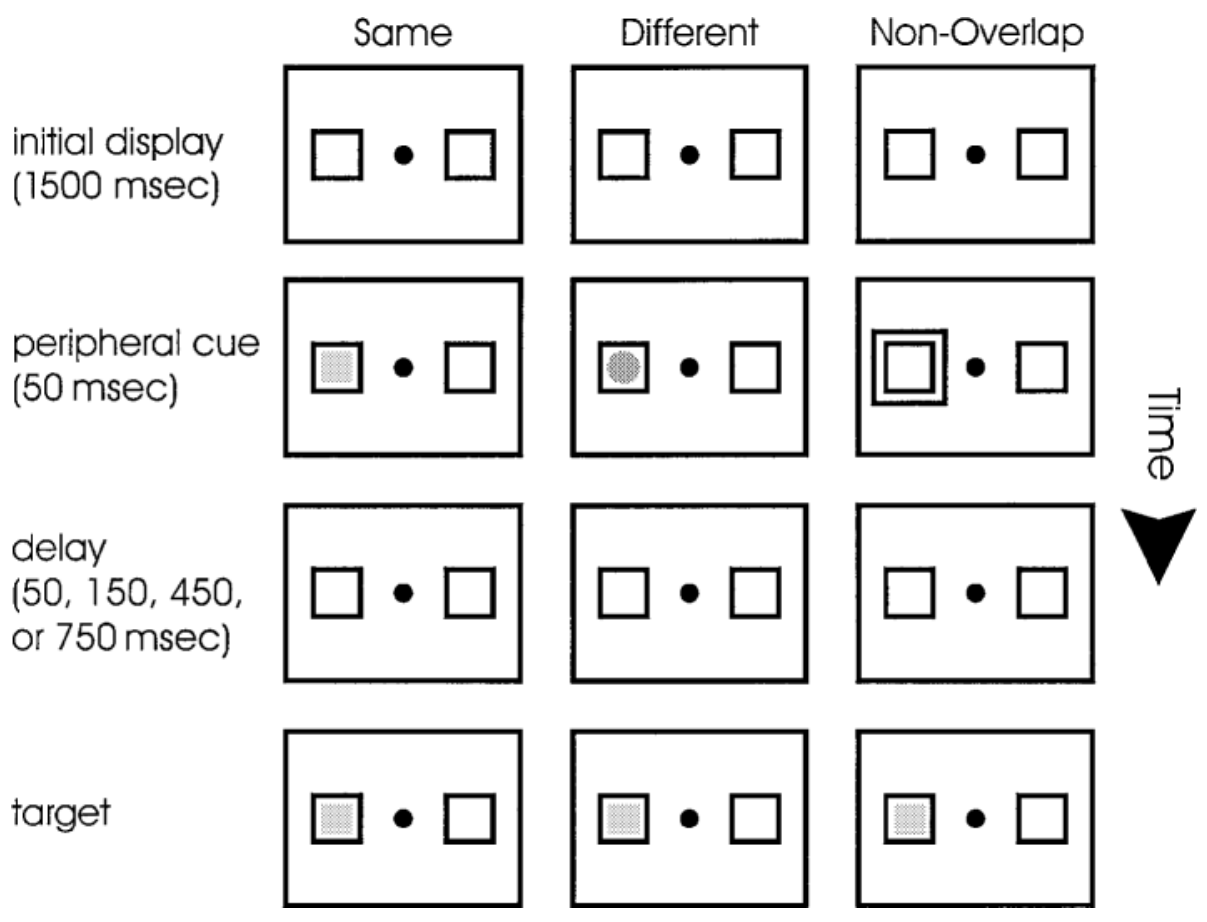

Figure 1. Trial sequence used in the experiment. In the different condition, the cue was green. The cue and target were equally likely to appear in either the left or the right box on any given trial. See text for details. 
Table 1

Mean Reaction Times (RTs) in Milliseconds for the Cued and Uncued Trials for Each Combination of Condition and SOAs (in Milliseconds)

\begin{tabular}{lccc}
\hline Condition & SOA & Cued RT & Uncued RT \\
\hline Same & 100 & 455 & 466 \\
& 200 & 437 & 436 \\
\multirow{5}{*}{ Different } & 400 & 424 & 396 \\
& 800 & 427 & 387 \\
& 100 & 438 & 435 \\
Nonoverlap & 200 & 419 & 405 \\
& 400 & 421 & 386 \\
& 800 & 411 & 376 \\
& 100 & 430 & 451 \\
& 200 & 399 & 425 \\
& 400 & 418 & 389 \\
\hline
\end{tabular}

tween condition and SOA were significant $[F(2,22)=$ $4.3, p<.03]$. Finally, the three-way interaction of condition, SOA, and trial type was significant $[F(6,66)=2.3$, $p<.05]$. No other main effects or interaction reached significance $(p>.5)$.

In order to understand the interaction effects, post hoc $t$ tests were performed on cued and uncued trials for each combination of condition and SOA. To aid in the interpretation of these analyses, Figure 2 shows the mean cuing effect (positive values indicate facilitatory cuing effects, negative values indicate IOR effects) for combination of condition and SOA. Turning to the same condition, there is a small, nonsignificant facilitation effect at the 100msec SOA (10 msec, $p>.10)$, virtually a null effect at the 200 -msec SOA (4 msec, $p>.40$ ), and inhibition at the 400 -msec SOA $(-28 \mathrm{msec}, p<.01)$ and 800 -msec SOA $(-40 \mathrm{msec}, p<.01)$. No facilitation was found in the different condition, with small nonsignificant inhibitory effects at the 100-msec SOA $(-10 \mathrm{msec}, p>.25)$ and 200msec SOA $(-14 \mathrm{msec}, p>.10)$ and large inhibitory effects at the $400-\mathrm{msec}$ SOA $(-35 \mathrm{msec}, p<.02)$ and $800-\mathrm{msec}$ SOA $(-35 \mathrm{msec}, p<.01)$. Only the nonoverlap condition produced the classic biphasic pattern of RTs, with significant facilitation at the $100-\mathrm{msec}$ SOA $(25 \mathrm{msec}$, $p<.01)$ and $200-\mathrm{msec}$ SOA $(27 \mathrm{msec}, p<.05)$ and significant inhibition at the $400-\mathrm{msec}$ SOA $(-29 \mathrm{msec}, p<$ $.01)$ and $800-\mathrm{msec}$ SOA $(-33 \mathrm{msec}, p<.01)$.

In order to examine the possibility of any speedaccuracy trade-offs between the conditions, errors were submitted to a $4($ SOA $) \times 2$ (trial type $) \times 3$ (cue) ANOVA. This analysis was conducted on the total number of errors and trimmed trials per condition because of the relatively rare occurrence of anomalous responses. Only the interaction of condition $\times$ SOA was significant $[F(6,66)=3.3, p<.01]$, with same condition producing the most errors at 100- and 800-msec SOAs, the different condition producing the most errors at the $400-\mathrm{msec}$ SOA, and the nonoverlap producing the most errors at the 200-msec SOA. There is no evidence of a speedaccuracy trade-off since the condition $\times$ SOA interaction was not significant for the RTs.

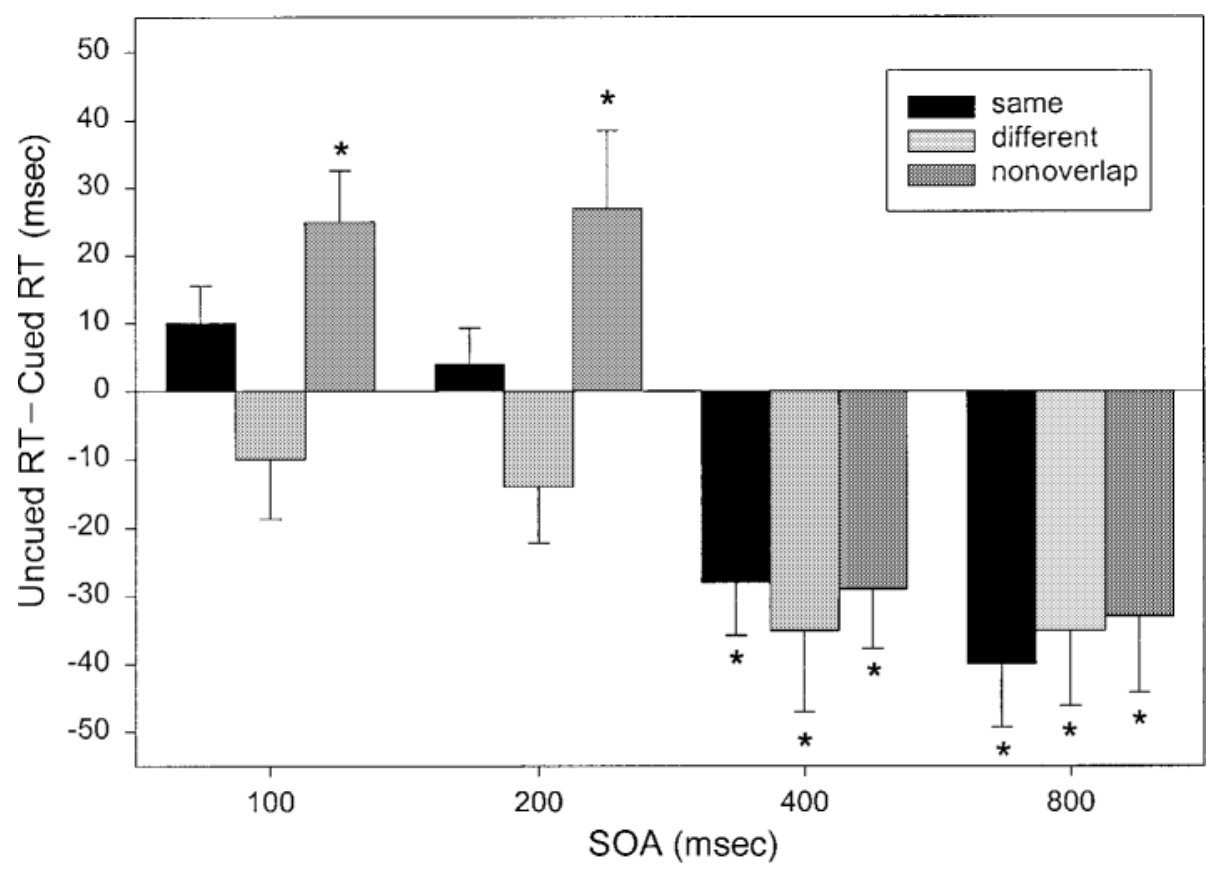

Figure 2. Mean cuing effect (uncued RT minus cued RT) for same, different, and nonoverlap conditions at each SOA. Error bars represent standard error, and asterisks indicate significant $(p<.05)$ cuing effects (positive values for facilitation, negative values for inhibition). 


\section{DISCUSSION}

Following the original work on IOR by Posner and Cohen (1984) and Maylor (1985), it has been generally thought that covertly attending to a peripheral cue will result in a brief period of facilitated target detection at the cued location followed by a longer period of inhibited target detection. The expectation of this biphasic pattern of early facilitation and late inhibition following a cue has led some researchers to suggest that attentional cuing effects must precede IOR (e.g., Taylor \& Klein, 1998a). More recently, Tassinari and his colleagues (e.g., Tassinari et al., 1994) have shown that the covert orienting of attention produces inhibitory effects at both short and long SOAs, although the magnitude of these effects are much larger at long SOAs. In attempting to explain these contradictory findings, Lupiáñez and Weaver (1998) have suggested that the early inhibition found by Tassinari et al. (1994) was due to cue-target discriminability. Because the same stimulus was used for both the cue and target by Tassinari et al. (1994), there may have been some confusion on cued trials as to the status (cue or target) of the target stimulus at the short SOA. Although this notion was rebutted by Tassinari et al. (1998), the possible effects of the physical characteristics of cues and targets in attentional cuing tasks remain unclear. Indeed, Maruff et al. (1999) have shown that the temporal characteristics of cues and targets play a role in determining whether or not early facilitation and later IOR will be found. The purpose of the present study was to determine if nontemporal stimulus characteristics also may affect the pattern of responses times found at various SOAs.

The findings of the present experiment are straightforward. All three conditions yielded IOR effects at the 400-msec and 800-msec SOAs, but only the nonoverlap condition yielded significant attentional cuing effects at the 100-msec and 200-msec SOAs. Rather, at the shorter SOAs, small and nonsignificant facilitatory (same condition) and inhibitory (different condition) effects were found. Thus, it appears that the degree of spatial overlap between cue and target does have a major effect on attentional cuing at short SOAs. It is worth noting that although the present findings indicate that one dimension (spatial overlap) is an important variable, cues and targets can differ along many other dimensions and along various degrees within a dimension. On the one hand, it may be that other combinations of color, shape, or luminance might produce sufficient cue-target discriminability for attentional cuing effects to be found. On the other hand, the results from the different condition also suggest that cues that are much more intense than the subsequent targets might be able to produce sufficient sensory masking to produce early occurring inhibition. It may also be that the absence of any spatial overlap between cues and targets, regardless of degree, yields early facilitation. Only by parametrically varying a wide number of dimensions over a wide range of degrees will a complete picture be found of what combination of cues and target will likely show early facilitatory effects. However, the present study clearly shows that cue-target discriminability is an important issue and spatial overlap is a potentially critical dimension.

The finding of IOR effects in all three conditions also indicates that all three types of cues were attended to, despite the fact that only the nonoverlap cue yielded early facilitatory effects. Thus, the present results suggest that IOR may be a good indicator of where attention was, although attentional cuing effects may not be a good indicator of where attention is. In other words, one's finding early facilitatory effects provides strong evidence of where attention is, whereas one's not finding such effects provides only weak evidence of where attention is not. IOR, being beyond the reach of most short-lived sensory effects, may be the best indicator of where attention was allocated in a visual display. The suggestion that IOR occurs at previously attended (i.e., cued) locations, even if those locations do not show attentional cuing effects, is consistent with the notion that IOR aids in visual searches and foraging behavior (e.g., Klein \& MacInnes, 1999).

It should be noted that, although the same and different conditions did not yield early facilitation, they also did not yield early inhibition effects, such as those found by Tassinari and Berlucchi (1993), who also used a short SOA of $200 \mathrm{msec}$. However, there are several methodological differences between the Tassinari and Berlucchi study and the present experiment. For example, Tassinari and Berlucchi used LEDs for cues and targets, along with very short cue and target presentations. More research will be needed to determine exactly what factor, or combination of factors, will consistently yield early inhibitory effects. It is also worth noting that the finding of an attentional cuing effect in the nonoverlap condition replicates the findings of Posner and Cohen (1984), who also used cues and targets that did not spatially overlap.

The present results provide further evidence that stimulus characteristics may differentially affect the pattern of detection responses at short and long SOAs (Maruff et al., 1999). This can be seen by the fact that the amount of spatial overlap between the cue and target, which played a critical role at the short SOAs, did not have any effect at the long SOAs. In other words, IOR appears to be unaffected by the spatial overlap of cues and targets. Thus, IOR may be relatively impervious to feature-based discriminability effects because cues and targets can be distinguished by their very salient temporal order. At short SOAs, the attention capturing properties of the cues may not be found in RTs because of other factors, one of which is presumably cue-target discriminability. By extension, IOR may be a better measure of attentional orienting when the effects of the specific physical characteristics of the stimuli are not known. This leads to the suggestion that IOR may indicate what locations were attended, even if no cuing effects at short SOAs were found at those locations. This is a complete reversal of the notion that attentional cuing effects must precede IOR (e.g., Maylor, 1985; Taylor \& Klein, 1998a). Of course, this notion is 
limited to situations in which IOR can be found (e.g., noninformative cues, long SOAs).

The notion that stimulus characteristics, such as the spatial (present study) and temporal (Maruff et al., 1999) overlap between cues and targets, have profound effects at short and long SOAs also indicates that the biphasic pattern of early facilitation and late inhibition should not be considered the definitive signature of the peripheral cuing paradigm. For example, this finding casts doubt on the assertion of Taylor and Klein (1998a) that the colorbased IOR effect found by Law, Pratt, and Abrams (1995) is not IOR, because Taylor and Klein (1998a) did not find facilitation at a short SOA. (It is worth noting that cues and targets did spatially overlap in Law et al. and in Taylor \& Klein, 1998a). Of course, it is possible to argue that the inhibitory effect found in the nonoverlap condition is IOR (due to the preceding attentional cuing effect), whereas the inhibitory effect found in the same and different conditions was some other effect (due to the lack of preceding attentional cuing effects). However, this seems unlikely given the almost identical magnitudes of inhibitory effects found in the three conditions. It should also be noted that the early facilitation found in the nonoverlap condition, without any temporal overlap between cue and target, is inconsistent with the findings of Maruff et al. (1999). There is no obvious reason for this discrepancy in results, although this does reinforce our contention of the importance that the physical characteristics of cues and targets play in the experimental methods used to examine the covert orienting of attention.

Finally, the finding of different attentional cuing effects, but similar IOR effects, with the three different types of cues leads to some possibilities about what occurs in a spatial cuing paradigm. One possibility is that, following the abrupt onset of the cue, attention will be oriented to the cued location, and this leads to the facilitation of detection responses. Also, following the onset of the cue, various sensory-based processes (e.g., cuetarget discriminability, masking) will become active. These processes tend to be of limited duration and tend to interfere with target detection. Thus, RTs at short SOAs will reflect both attentional orienting effects as well as the sensory effects associated with the stimuli. In situations in which the cue is offset several hundred milliseconds before the appearance of the target, only inhibitory attentional effects (i.e., IOR) will be present.

Another possibility is that cues may initiate independent facilitatory and inhibitory effects and that RTs are due to the interaction of the two effects. Depending on the relationship between the features of the cues and targets, a specific peripheral cue might have a variety of effects over a period of time. Extrapolating from the present experiment, it is possible that peripheral cues that are much more intense than subsequent targets tend to have greater inhibitory effects than facilitatory effects over a larger period of time, with the discrepancy between the effects being smallest at short SOAs. Support for this notion comes from Tipper, Weaver, and Houghton's (1994) conclusion that stimuli can contain both excitatory and inhibitory properties and that current goal states can determine which property will guide attentional selection.

At the moment, it is not possible to determine which of the two possibilities offers the best explanation for the present results. In fact, it is likely that sensory effects would interact with both the facilitatory and inhibitory effects of the cue. What is clear is that, although the present study examined spatial overlap between cues and targets and Maruff et al. (1999) examined temporal overlap, there are likely to be many stimulus characteristics that have a direct impact on detection RTs. Thus, the physical characteristics of the cues and targets must be considered before any conclusion can be safely reached regarding the orienting of attention in a spatial cuing paradigm.

\section{REFERENCES}

Danzinger, S., Kingstone, A., \& Snyder, J. J. (1998). Inhibition of return to successively stimulated locations in a sequential visual search paradigm. Journal of Experimental Psychology: Human Perception \& Performance, 24, 1467-1475.

Egly, R, \& Homa, D. (1991). Reallocation of visual attention. Journal of Experimental Psychology: Human Perception \& Performance, 17, 142-159.

Enns, J. T., \& Richards, J. C. (1997). Visual attentional orienting in developing hockey players. Journal of Experimental Child Psychology, 64, 255-275.

Folk, C. L., Remington, R. W., \& Johnston, J. C. (1992). Involuntary covert orienting is contingent on attentional control settings. Journal of Experimental Psychology: Human Perception \& Performance, 18, 1030-1044.

KLeIn, R. M., \& MacInnes, W. J. (1999). Inhibition of return is a foraging facilitator in visual search. Psychological Science, 10, 346-352.

Law, M. B., Pratt, J., \& ABrams, R. A. (1995). Color-based inhibition of return. Perception \& Psychophysics, 57, 402-408.

Lupiáñez, J., Milán, E. G., Tornay, F. J., Madrid, E., \& Tudela, P. (1997). Does IOR occur in discrimination tasks? Yes, it does, but later. Perception \& Psychophysics, 59, 1241-1254.

LUPIÁÑEZ, J., \& WEAVER, B. (1998). On the time course of exogenous cueing effects: A commentary on Tassinari et al. (1994). Vision Research, 38, 1621-1623.

Maruff, P., Yucel, M., Dankert, J., Stuart, G., \& Currie, J. (1999). Facilitation and inhibition arising from the exogenous orienting of covert attention depends on the temporal properties of spatial cues and targets. Neuropsychologia, 37, 731-744.

MAYLOR, E. (1985). Facilitatory and inhibitory components of orienting in visual space. In M. I. Posner \& O. S. M. Marin (Eds.), Attention and performance XI (pp. 189-304). Hillsdale NJ: Erlbaum.

Posner, M. I. (1980). Orienting of attention. Quarterly Journal of Experimental Psychology, 32, 3-25.

Posner, M. I., \& CoHEN, Y. (1984). Components of visual orienting. In H. Bouma \& D. G. Bouwhuis (Eds.), Attention and performance X (pp. 531-556). Hillsdale, NJ: Erlbaum.

Pratt, J. (1995). Inhibition of return in a discrimination task. Psychonomic Bulletin \& Review, 2, 117-120.

Pratt, J., \& Abrams, R. A. (1995). Inhibition of return to successively cued spatial locations. Journal of Experimental Psychology: Human Perception \& Performance, 21, 1343-1353.

Pratt, J., \& Aвrams, R. A. (1999). Inhibition of return in discrimination tasks. Journal of Experimental Psychology: Human Perception \& Performance, 25, 1343-1353.

Rafal, R. D., Calabresi, P. A., Brennan, C. W., \& Sciolto, T. K. (1989). Saccade preparation inhibits reorienting to recently attended locations. Journal of Experimental Psychology: Human Perception \& Performance, 15, 673-685.

Tassinari, G., Aglioti, S., Chelazzi, L., Marzi, C. A., \& Berluc- 
CHI, G. (1987). Distribution in the visual field of the costs of voluntarily allocated attention and of the inhibitory after-effects of covert orienting. Neuropsychologia, 25, 55-72.

Tassinari, G., Agliotti, S., Chelazzi, L., Peru, A., \& Berlucchi, G. (1994). Do peripheral non-informative cues induce early facilitation of target detection? Vision Research, 34, 179-189.

Tassinari, G., Agliotti, S., Chelazzi, L., Peru, A., \& Berlucchi, G. (1998). On the time course of exogenous cueing effects: A response to Lupiáñez and Weaver. Vision Research, 38, 1625-1628.

TASSINARI, G., \& Berlucchi, G. (1993). Sensory and attentional components of slowing of manual reaction time to non-fixated visual targets by ipsilateral primes. Vision Research, 33, 1525-1534.

TAY LOR, T. L., \& KLEIN, R. M. (1998a). Inhibition of return to color: A replication and nonextension of Law, Pratt, and Abrams (1995). Perception \& Psychophysics, 60, 1452-1456.

TAY LOR, T. L., \& KleIN, R. M. (1998b). On the causes and effects of inhibition of return. Psychonomic Bulletin \& Review, 5, 625-643.
Tipper, S. P., Weaver, B., \& Houghton, G. (1994). Behavioural goals determine inhibitory mechanisms of selective attention. Quarterly Journal of Experimental Psychology, 47A, 809-840.

Tipper, S. P., Weaver, B., Jerreat, L. M., \& Burak, A. L. (1994). Objectbased and environment-based inhibition of return of visual attention. Journal of Experimental Psychology: Human Perception \& Performance, 20, 478-499.

\section{NOTE}

1. Although this is the most common explanation for IOR, it is not the only explanation. For a detailed review of an oculomotor system explanation of IOR, see Taylor and Klein (1998b).

(Manuscript received October 12, 1999;

revision accepted for publication October 17, 2000.) 